\title{
EYEING THE HUMAN BRAIN'S SEGMENTATION METHODS
}

\author{
LILIAN CHIRU KAWALA ${ }^{1}$, XUEWEN DING ${ }^{1} \&$ GUOJUN DONG ${ }^{2}$ \\ ${ }^{l}$ Department of Signal and Information Processing, School of Electronic Engineering, \\ Tianjin University of Technology and Education, Tianjin, China \\ ${ }^{2}$ Tianjin Key Laboratory of High-Speed Railway Wireless Communication Enterprise, Tianjin, China
}

\begin{abstract}
Image segmentation (IS) is often the first and most important step in medical image analysis. Among other uses, it helps us perform the following tasks in images: to measure and visualize the anatomical structures of the brain; to highlight brain structural changes; and to delineate regions with severe pathology. Thus, it becomes much more significant in image-guided surgical planning. To date, a considerable body of research has burgeoned, which focuses on improving segmentation accuracy and reducing complexity. In the current study, we review well-selected articles describing the most popular segmentation methods commonly used in MRI studies. We mainly focus on highlighting the differences between these available methods, review their capabilities and advantages, and discuss their limitations and challenges. Conclusively, the study provides guidelines and directions for the appropriate segmentation approach(s) reliable and succinctly suite for MRI brain Images with good prospects. In order to fully understand concepts and problems behind segmentation methods, a need of thorough elaboration of segmentation perception is noteworthy. To do so, we have extensively established the most augmented introduction of basic segmentation concepts, and deeply explained the fundamental differences of brain-MRI preprocessing pipelines.
\end{abstract}

KEYWORDS: Segmentation, MRI-Scans, Multi-Atlases, Convolution \& Neural Network

Received: Mar 07, 2019; Accepted: Mar 27, 2019; Published: Apr 19, 2019; Paper Id: IJEEERJUN20195

\section{INTRODUCTION}

Segmentation of MRI-brain scans have played a key role in many studies in recent years. The main purpose of brain segmentation is localizing each unique brain structure based on tissue characteristics. With a fully-segmented brain, morphological information such as volume and shape, can be captured and used for future statistical analysis. The information helps researchers investigate how the brain is vulnerably affected by a neurological disease (see figure. 1). Most methods have previously been developed to solve the problem of brain segmentation.

The intensity in MRI data offers a fundamental information in the segmentation process in most of these methods. Despite the intensity information being useful, the resulting segmentation is inaccurate due to noise, nonuniformity of intensity and poor image contrast in the MR data. Although MRI-data preprocessing pipeline involves processes that aim at reducing noise and artefacts, there is no guarantee that the structure and tissue intensities are unique as claimed priority. On the other hand, structures such as hippocampus and amygdala, for example, belong to gray matter, with almost identical intensities which makes difficult to differentiate them using the intensity aspect alone[1]. 


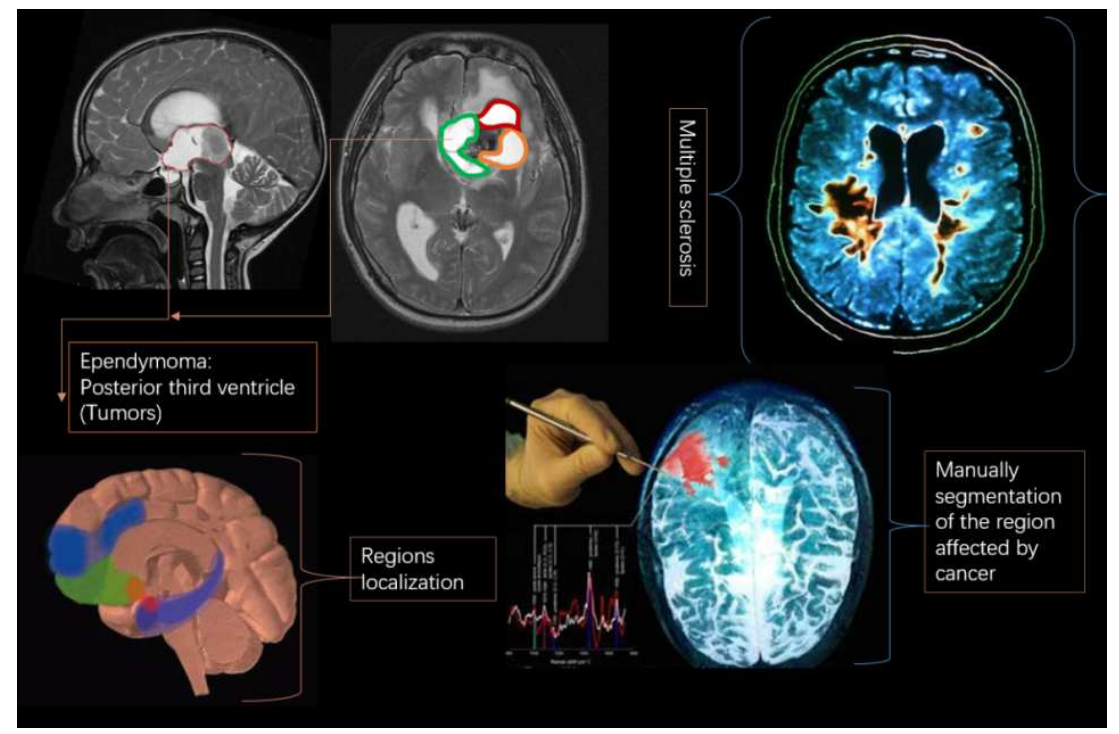

Figure 1: Segmentation in Different Purposes: Delineate Multiple Sclerosis, Tumors, Cancers and Brain Regions Partitioning

Consequently, brain segmentation accuracy would not be sufficient enough to perform meaningful statistical analysis.

To increase the accuracy and robustness, algorithms have been developed to not only use the brain intensity information but also incorporate spatial and contextual information. The spatial pattern characteristic of the brain structure is well known even prior to MRI scanning. The information can easily enable locating amygdala as always anterior and superior to hippocampus. Studies have also identified that brain tissues often appear in clusters matter voxels, for instance, are generally surrounded by more gray matter voxels. Using this information seems to confidently be possible to incorporate spatial and contextual information for improved segmentation [2].

One among the methods that takes advantage of this direction is graphical models. In graphical models, the probability of each voxel-label given the current estimate of the labels in a finite number of neighboring voxels is computed while tuning various hyper parameters. Other techniques rely on tissue probability maps and manually segmented structural atlases of the particular image.

\section{MRI-Image Preprocessing Concepts}

Automated analysis of MR images is challenging due to intensity in homogeneity, variability of the intensity ranges and contrast, and noise. Therefore, prior to automated analysis, certain steps are required to make the images appear more similar, and these steps are commonly referred to as preprocessing.

Typical preprocessing steps for structural brain MRI include the following key steps. 


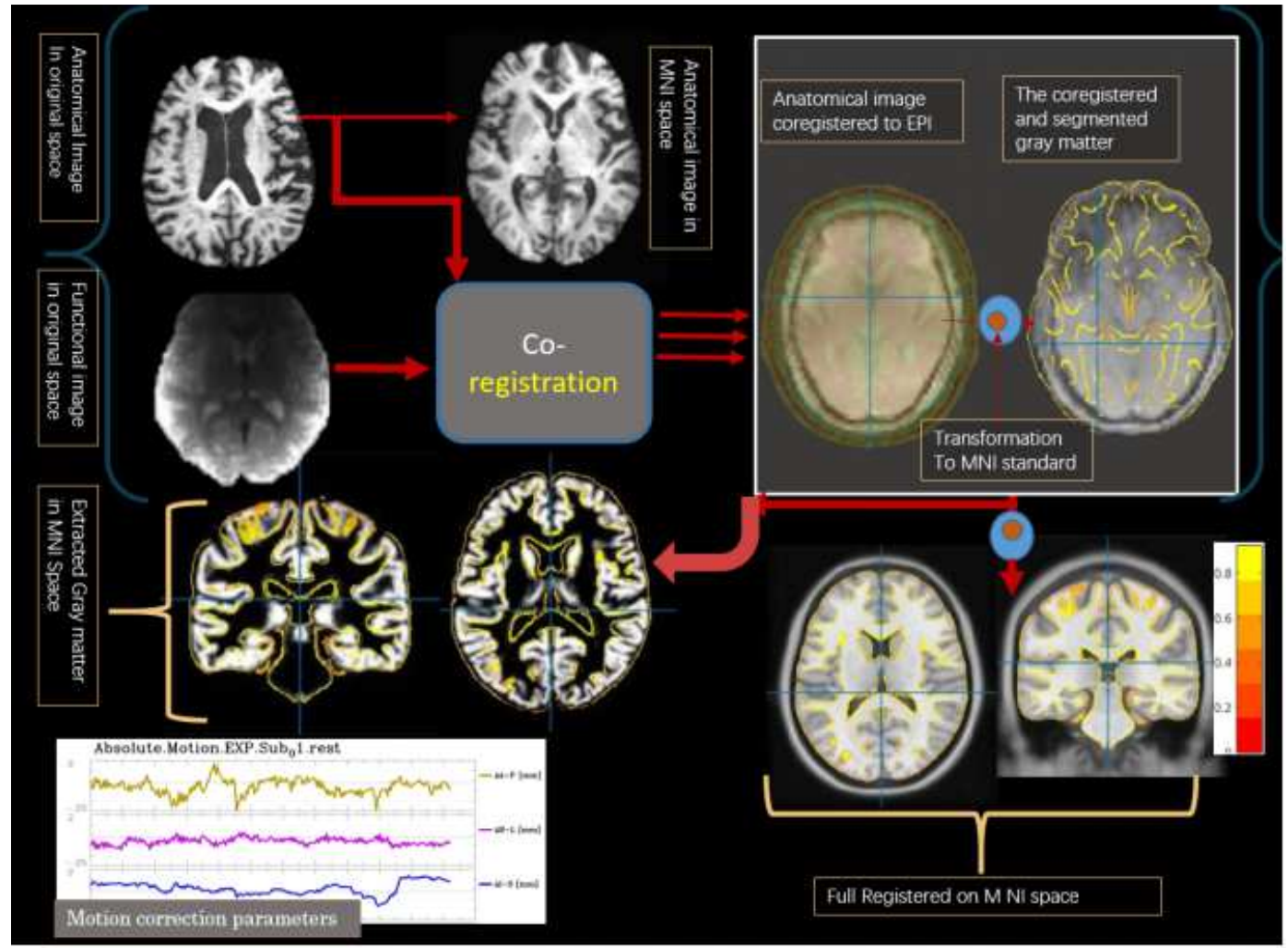

Figure 2: Segmentation Pipeline

\section{Image Registration Perspective}

Traditionally, the preprocessing begins with image registration. In image registration, two or more images with similar contents taken in regular interval (TR) or different viewpoints are overlaid (spatially aligned). More information about the patients' health can be thoroughly extracted with multi-modal image registration, i.e., MRI, CT, PET, and SPECT. The obtained information plays a decisive role in describing the extent of pathology. The most common type of registration in preprocessing pipeline is co registration sometimes referred to intra-subject registration. Under this, single subjects images acquired from two or more modalities are spatially realigned (see figure 2)

The more specific and practical scenario is co-registration involving anatomical image, i.e., T1 weighted images, and functional (fMRI) images with the purpose of correcting for motion. Its use can be extended to spatial space standard normalization which is a potential step in the MRI cross-sectional study [3]

\section{SEGMENTATION METHODS}

Diverse applications in medical imaging have led to the development of various segmentation techniques. Many have claimed that no single method can be suitable for all types of images nor are all methods equally good for a particular type of image processing pipeline. However, it has recently been vindicated that a single approach designed for a certain images class could be easily extended to another class. Therefore, we opt to explore in depth these available segmentation methods and critically analyze their limitations to help suggest an appropriate direction for the stable segmentation method. Our hypothesis stands to argue that the feasible segmentation method could be available or developed that would be adjustable to fit various preprocessing pipelines for divergent applications [4]. 


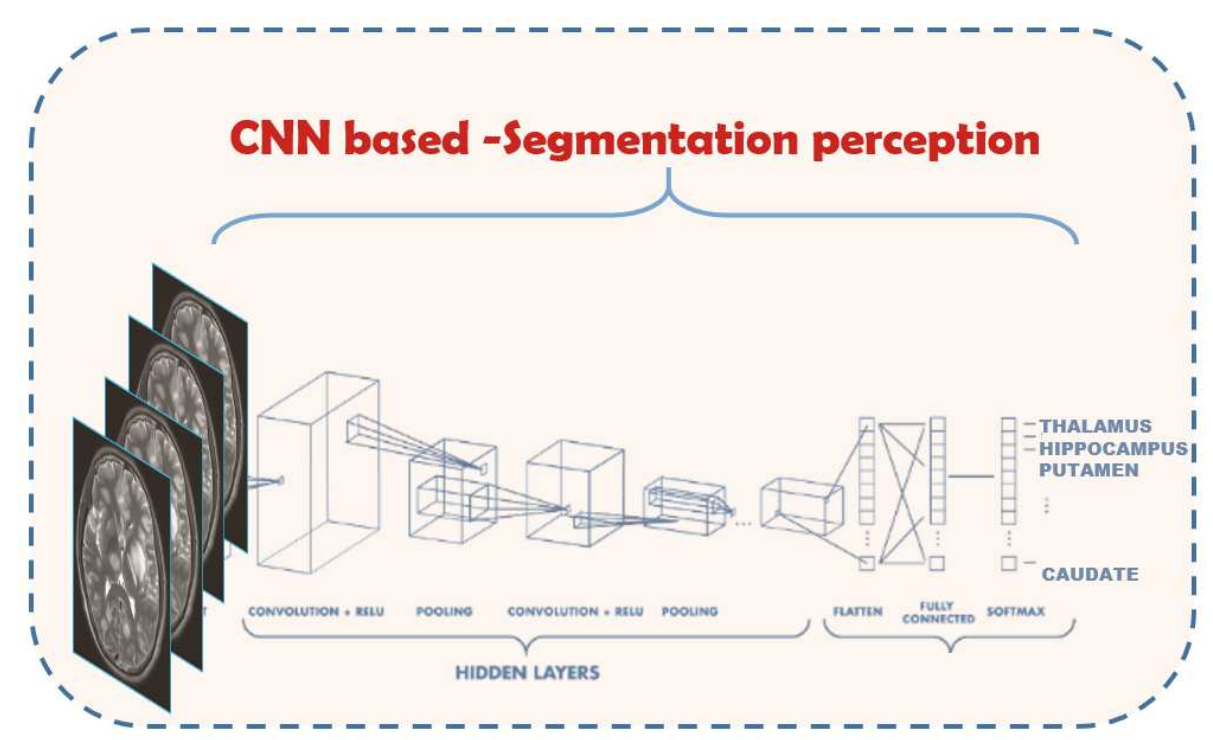

Figure 3: CNN Based-Segmentation

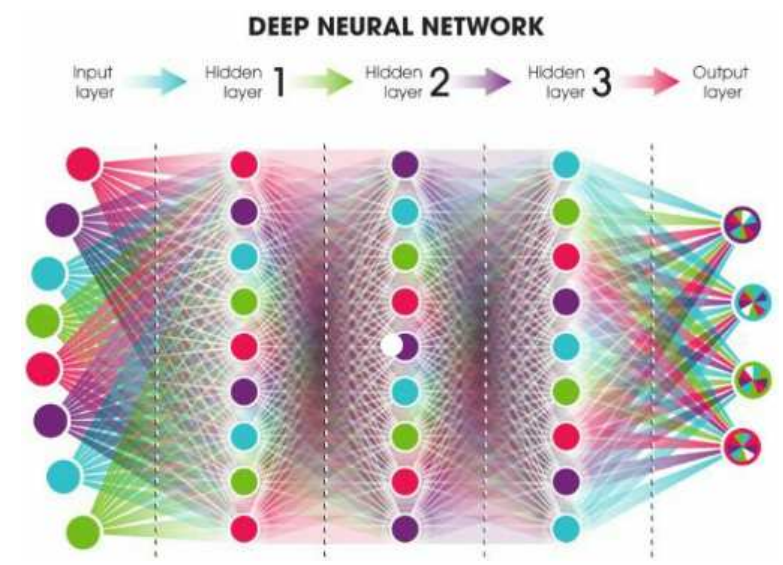

Figure 4: Broader View of Deep Learning

\section{Deformation-Based Morphometry}

Deformation-based morphometry (DBM) gained confidence and popularity through its efficiency in identifying global and gross brain volume changes. It is capable of characterizing volumes and shapes of the brain across different population. The DBM heavily relies on deformation generated by nonlinear registration with a brain template.

The resulting deformation field contains useful information about local volume and shape of the patients' brain. To recover this information, the Jacobian determinant, also known as the volume expansion factor, is applied to the deformation field. The Jacobian, in theory, describes the amount of stretching, rotating and shearing that the non-linear transformation has imposed on MR scan. With the determinant applied to the Jacobian, local brain growth or shrinkage, relative to the brain template, is revealed. In particular, the Jacobian determinant greater than one (JD>1) signifies volumetric expansion, while that less than one $(\mathrm{JD}<1)$ is associated with local shrinkage. When the value is one $(\mathrm{JD}=1)$, it suggests that there is no volume change.

When many subjects scans are aligned to a common anatomical space, statistical models can be applied across the Jacobian determinant of the deformation fields, on a voxel-wise basis, to identify localized group volumetric differences $[6,7]$. 


\section{Voxel-Based Morphometry}

In contrast to DBM, voxel-based morphometry (VBM) detects regional brain volume changes at the mesoscopic scale, i.e., several millimeters. Identification of regional tissue volume differences of gray matter, white matter, and CSF is absolutely achieved in VBM. It is desirable to use DBM and VBM together because they complement each other by capturing brain volume changes at both the macroscopic and mesoscopic scales.

Initially, MR scans are linearly transformed into a common anatomical space. This is followed by tissue classification, where each voxel will be assigned one of four classes: gray matter, white matter, CSF, or non-brain tissue. The resulting tissue maps are then blurred with a Gaussian kernel. Blurring is an important stage in VBM because it helps facilitate non-linear registration, performed later, by providing a better capture range for local displacements and mitigating registration errors. Blurring also permits Gaussian assumptions to be applied in later statistical analysis. If these tissue maps were not blurred, then complex discrete statistics would be required. The resulting blurred tissue maps are referred to as tissue density maps, which reflect the local concentration of each respective tissue.

Finally, each tissue density map is non-linearly registered its corresponding tissue probability map, to account for global brain shape differences. Statistical analysis can then be performed on a voxel wise basis across the entire population to determine regional tissue volume differences. VBM can also incorporate a modulation step where the Jacobian determinant of the deformation field from the estimated non-linear transformation are multiplied with each voxel. By doing so, it will preserve the volume of particular tissues that may have been expanded or contracted during non-linear registration. When the modulation step is included the technique is referred to as optimized VB $[8,9,10]$.

\section{Multi-Atlas Registration Based Method}

Generally segmentation methods rely on intensity information and higher order structural information of objects. The prominent example is Multi-Atlas registration method, which is based on a specific set of templates whereby in a medical application, typically contains labelling of anatomical structures.

The field of brain segmentation is dominated by multi-atlas based methods. They are driven by a combination of spatial normalization through registration followed by a classification step, which can be a simple majority voting. If an atlas or templates of the human brain for a specific population of interest are available, then atlas-based method can be a powerful tool for brain MRI segmentation. The main advantage of these methods is the possibility to segment any brain structure available in the atlas without any additional cost. Therefore atlas-based approaches are similar to classify methods, except that they are implemented in spatial domain rather than in the feature space.

The alignment of a probabilistic atlas with the image to be segmented, will ensure the prior knowledge of the method. Since the segmentation labels and the ground truth are known for the atlas, all atlas information is transferred to the target image after registration. Therefore, the performance of atlas-based methods are directly dependent on the quality of the registration method used.

However the brain extraction using a probabilistic atlas is usually not very accurate and can cause misclassification around the brain boundary. The common way of aligning the probabilistic atlas with the image is to use affine registration. However if brain anatomy of interest differs from the probabilistic atlas an affine alignment may not be sufficient. Therefore suggest aligning the atlas using non rigid registration. But in other literature shows difficulties in registering anatomical template with the image to be segmented using standard registration methods. In addition, even with 
non-rigid registration methods, accurate segmentation of a complex structure is difficult due to anatomical variability.

Atlas guided segmentation in patients with brain deformations can be difficult and prone to errors, because the probabilistic atlas is based on a population of healthy subjects. Regardless of the type of atlas-based segmentation (single or multi) or the atlases used, the accuracy of segmentation is highly dependent on the registration methods utilized $[11,12,13,14]$.

\section{Markov Random Field Models}

MRF modelling itself is not a segmentation method, but a statistical model that can be used within segmentation methods. MRFs model spatial interactions between neighbouring or nearby pixels. These local correlations provide a mechanism for modelling a variety of image properties (60).

In medical imaging, they are typically used because most pixels belong to the same class as their neighbouring pixels. In physical terms, this implies that any anatomical structure that consists of only one pixel has a very low probability of occurring under an MRF assumption. MRFs are often incorporated into clustering segmentation algorithms such as the $\mathrm{K}$-means algorithm under a Bayesian prior model.

The segmentation is then obtained by maximizing the a posteriori probability of the segmentation, given the image data. This maximization can be achieved by iterative methods such as iterated conditional modes or simulated annealing. A difficulty associated with MRF models is proper selection of the parameters controlling the strength of spatial interactions. A setting that is too high can result in an excessively smooth segmentation and a loss of important structural details. In addition to that, MRF methods usually require computationally intensive algorithms. Despite these disadvantages, MRFs are widely used not only to model segmentation classes, but also to model intensity in homogeneities that can occur in MR images and textural properties, which is useful in the segmentation of digital mammograms $[15,17,18,19,20]$.

\section{Region Growing Method}

Region growing is a technique for extracting a connected region of the image which consists of groups of pixels/voxels with similar intensities. In its simplest form, region growing starts with a seed point (pixel/voxel) that belongs to the object of interest. The seed point can be manually selected by an operator or automatically initialized with a seed finding algorithm. Then, region growing examines all neighboring pixels/voxels and if their intensities are similar enough (satisfying a predefined uniformity or homogeneity criterion), they are added to the growing region.

This procedure is repeated until no more pixels/voxels can be added to the region. Region growing is suitable for segmentation of volumetric images which are composed of large connected homogeneous regions. Thus, it is successfully used in medical image analysis to segment different tissues, organs, or lesions from MR images. For example, it is used in the brain MRI analysis for segmentation of brain vessels, brain tumour segmentation, or extraction of brain surface.

The primary disadvantage of region growing is that it requires manual interaction to obtain the seed point. Thus, for each region that needs to be extracted, a seed must be planted. Split and-merge is an algorithm related to region growing, but it does not require a seed point. Region growing can also be sensitive to noise, causing extracted regions to have holes or even become disconnected. Conversely, partial volume effects can cause separate regions to become connected. To help alleviate these problems, a homo-topic region-growing algorithm has been proposed that preserves the 
topology between an initial region and an extracted region [ 21, 22, 23, 24, 25].

\section{Convolution Neural Network}

In recent years, a growing interest has been drawn to the Convolution Neural Network. Having a remarkable performance in classification and detection, researchers seem to extend its use in the segmentation tasks. The need of alternative approach arises due to the fact that the images accumulated from imaging modalities depict divergent morphological patterns of the brain structures. The rationale is linked to variations in imaging parameter settings.

Therefore, Deep learning is said to be effective in exploiting spatial structures of images by utilizing local features. Through feature descriptors, Deep learning provides distinct characteristics potential for segmentation unlike the previous methods. A typical example of $\mathrm{CCN}$ is given in fig. 3. Other researchers argue that CNN capability exceeds human imagination as it is believed that even lowest contrast pathological features can be captured by this approach; making it suggested for many clinical applications.

Although the method becomes a future hope, there are few challenges to be underlined associated to CNN lone, CNN depends on massive data to promise a good result which seem to be a problem clinically because most of clinical cases consist of limited data set unlike common natural images; second, Medical images exhibit 3D high resolution, seeking models with high dimension when dealing with them contrary to natural images.

Nevertheless, a good news in computer vision technology inform us about advancement in Graphic Processing Unit, which accelerates computing speed and thus, allowing fast training of data on a complex deep networks while reducing top 5 error(from $26.2 \%$ to $15.3 \%$ ).

Through several trials, $\mathrm{CNN}$ has been proven to provide optimal solution in segmentation tasks with efficiency to handle bias and variance (Over fitting and under fitting). Along with such success, more other fields present magnificent achievements with deep learning: object detection and disease classification. Due to the adaptive utilization of deep learning, different form/models of DL exist, such as deep neural networks, stacked auto-encoders, deep Boltzmann machines, and convolution neural networks (CNNs) [ 26, 27, 28, 29, 31, 32].

\section{CONCLUSIONS}

Among the aforementioned methods, CNN gives more promising result, and is a significantly powerful tool for segmentation. The future direction will be totally dependent of the CNN-based approaches.

The intensity based methods gain no confidence because several regions of the brain consist of similar intensities, making it difficult for segmentation of the regions. The deformation-based morphometry methods fail to capture the intermediate range of features since the Jacobian determinant does not describe the value between the ranges; they threshold the values and leave other intermediate values unaccountable.

The Multi-Atlas Registration Based Methods are currently working better on the field, but suffer from parameter estimations and criteria. Many data are reported to be discarded because they give bad segmentation results.

The Markov Random Field Models provide approximately better results, but involves several approximations and assumptions. The region growing methods are efficient in other applications, but not useful in medical images since can over estimate or underestimate the pathology during the process. In this, regard, despite the challenging of a large dataset, CNN-based methods remain the future hope for proper segmentation with account to many challenging pinpointed in other 
methods. The CCN-based methods are robust to brain structural changes and motion.

\section{REFERENCES}

1. Brummer, M. E. (1992). Optimized intensity thresholds for volumetric analysis of magnetic resonance imaging data. Proc. SPIE, 1808, 299-310

2. Zhang, Y., Brady, M., \& Smith, S. (2001). Segmentation of Brain MR Images through a Hidden Markov Random Field Model and the Expectation Maximization Algorithm. IEEE Transactions on Medical Imaging, 20(1)

3. Zijdenbos, A. P., Forghani, R., \& Evans, A. C. (2002). Automatic 'pipeline' analysis of 3-D MRI data for clinical trials: application to multiple sclerosis. IEEE Transactions on Medical Imaging, 21(10), 1280-1291.

4. N. R. Pal and S. K. Pal, review on image segmentation techniques, Pattern Recognition, vol. 26, no. 9, pp. 1277294, 1993

5. M. Prastawa, An MRI segmentation framework for brains with anatomical deviations [Ph.D. thesis], University of North Carolina at Chapel Hill, 2007. Cross-sectional MRI data in young, middle aged, nondemented, and demented older adults. Journal of Cognitive Neuroscience, 19(9), 1498-1507

6. Thompson, P. M., \& Toga, A. W. (1999). Anatomically driven strategies for high-dimensional brain image warping and pathology detection. Brain Warping, 311-336.

7. L Chung, M. K., Worsley, K. J., Paus, T., Cherif, C., Collins, D. L., Giedd, J. N., Evans, A. C. (2001). A Unified Statistical Approach to Deformation-Based Morphometry. NeuroImage, 14(3), 595-606.

8. Good, C. D., Johnsrude, I. S., Ashburner, J., Henson, R. N., Friston, K. J., \& Frackowiak, R. S. (2001). A Voxel-Based Morphometric Study of Ageing in 465 Normal Adult Human Brains. NeuroImage, 14(1), 21-36.

9. Collins, D. L., Holmes, C. J., Peters, T. M., \& Evans, A. C. (1995). Automatic 3-D Model-Based Neuroanatomical Segmentation. Human Brain Mapping, 3(3), 190-208.

10. Ashburner, J., \& Friston, K. J. (2000). Voxel-Based Morphometry - The Methods. NeuroImage,11(6), 805-821

11. E. D'Agostino, F. Maes, D. Vandermeulen, and P. Suetens, viscous fluid model for multimodal nonrigid image registration using mutual information, in Proceedings of the Medical Image Computing and Computer Assisted Intervention (MICCAI '02), pp. 54148, 2002.

12. D. Rueckert, L. I. Sonoda, C. Hayes, D. L. Hill, M. O. Leach, and D. J. Hawkes, on rigid registration using free-form deformations: application to breast images, IEEE Transactions on Medical Imaging, vol. 18, no. 8, pp. 71221, 1999.

13. N. I. Weisenfeld and S. K. Warfield, Automatic segmentation of newborn brain MRI, NeuroImage, vol. 47, no. 2, pp. 56472, 2009.

14. M. Kuklisova-Murgasova, P. Aljabar, L. Srinivasan et al., dynamic 4D probabilistic atlas of the developing brain, NeuroImage, vol. 54, no. 4, pp. 2750763, 2011.

15. K. Held, E. R. Kops, B. J. Krause, W. M. Wells, and R. Kikinis, Markov random field segmentation of brain MR images, IEEE Trans. Med. Imag., vol. 16, pp. 878886, Dec. 1997.

16. Venugopal, N., \& Dongre, M. A Novel Technique that Maximizes Ergodic and Outage Capacity for Enhanced Spectrum Sharing in Cognitive Radio Network.

17. L. R. Rabiner, A tutorial on hidden Markov models and selected applications in speech recognition, Proc. IEEE, vol. 77, pp. 257286, Feb. 1989. 
18. A. Kundu, Y. He, and P. Bahl, Recognition of handwritten word: First and second-order hidden Markov model based approach, Pattern Recogn., vol. 22, no. 3, pp. 283297, 1989.

19. J. Zhang, The mean field theory in EM procedures for blind Markov random field image restoration, IEEE Trans. Image Processing, vol. 2, pp. 2740, Jan. 1993.

20. S. Z. Li, Markov Random Field Modeling in Computer Vision. Berlin, Germany: Springer-Verlag, 1995.

21. R. M. Haralick and L. G. Shapiro, Image segmentation techniques, Computer Vision, Graphics, and Image Processing, vol. 29, no. 1, pp. 100132, 1985.

22. N. Passat, C. Ronse, J. Baruthio, J.-P. Armspach, C. Maillot, and C. Jahn, Region-growing segmentation of brain vessels: an atlas-based automatic approach, Journal of Magnetic Resonance Imaging, vol. 21, no. 6, pp. 715725, 2005.

23. T. Weglinski and A. Fabijanska, Brain tumor segmentation from MRI data sets using region growing approach, in Proceedings of the 7th International Conference on Perspective Technologies and Methods in MEMS Design (MEMSTECH'11), pp. 185188, May 2011.

24. M. del Fresno, M. Vnere, and A. Clausse, A combined region growing and deformable model method for extraction of closed surfaces in 3D CT and MRI scans, Computerized Medical Imaging and Graphics, vol. 33, no. 5, pp. 369376, 2009.

25. Manousakas IN, Undrill PE, Cameron GG, Redpath TW. 1998. Split-and-merge segmentation of magnetic resonance medical images: performance evaluation and extension to three dimensions. Comput. Biomed. Res. 31:393412.

26. Deng J, et al.: BImageNet: A large-scale hierarchical image database, in 2009 I.E. Conference on Computer Vision and Pattern Recognition, 2009.

27. O. Russakovsky et al., BImageNet large scale visual recognition challenge, Int. J. Comput. Vis., vol. 115, no. 3, pp. 21152, 2015 .

28. Krizhevsky A, Sutskever I, Hinton GE: ImageNet classification with deep convolutional neural networks. In: Pereira F, Burges CJC, Bottou L, Weinberger KQ Eds. Advances in neural information processing systems 25. USA: Curran Associates, Inc., 2012, pp. 1097105.

29. B. Fischl, D. Salat, E. Busa, M. Albert, M. Dieterich, C. Haselgrove, A. van der Kouwe, R. Killiany, D. Kennedy, S. Klaveness, A. Montillo, N. Makris, B. Rosen, and A. Dale. Whole brain segmentation: automated labeling of neuroanatomical structures in the human brain. Neuron, 33:34155, 2002.

30. Alasadi, A. H. H., Mohammed, H., \& Alshemmary, E. N. (2013). Hybrid k-means Clustering for Color Image Segmentation. Science and Engineering (IJCSE), 2(4), 17-26.

31. P. Coupe, J. Manjon, V. Fonov, J. Pruessner, M. Robles, and D. Collins. Patch-based segmentation using expert priors: application to hippocampus and ventricle segmentation. NeuroImage,54(2):94054, 2011.

32. A. Brebisson and G. Montana. Deep neural networks for anatomical brain segmentation. In Proceedings of the IEEE Conference on Computer Vision and Pattern Recognition Workshops, pages208, 2015. 
